El Computador como Modo de Presentación de la Lectura en Inglés. Universidad de Nariño, Editorial Universitaria CEPUN. San Juan de Pasto. ISBN: 978-958-9479-12-4. 1998.

APA citation style: Benavides B., J. E. (1998). El Computador como Modo de Presentación de la Lectura en Inglés: Efectos del Modo de Presentación de Texto en el Computador en la Velocidad y Comprensión de la Lectura en Inglés en Estudiantes de la Universidad de Nariño. San Juan de Pasto: Universidad de Nariño, CEPUN.

\title{
El Computador como Modo de Presentación de la Lectura en Inglés
}

\author{
Jorge E. Benavides B. (joelbebu@gmail.com) \\ Departamento de Lingüística e Idiomas \\ Universidad de Nariño, San Juan de Pasto, Colombia
}

\section{Revisión de la literatura}

\subsection{Introducción}

Ha existido mucha especulación acerca del uso del computador en educación durante los últimos veinte años. Se han hecho bastantes afirmaciones sobre las ventajas del computador en el salón de clases (Kinzer, 1986) como la del incremento de la motivación y la atención que este puede ofrecer a los estudiantes (White, 1983).

A pesar de que se han realizado algunos tipos de estudios en este campo en forma incipiente, la mayoría de la evidencia acerca de la utilidad del computador ha sido de tipo anecdótico. Y a pesar de que la opinión de los expertos y los resultados de los estudios parecen apoyar y alentar el uso del computador en la enseñanza o instrucción de la lectura, pocos estudios se han completado sobre la instrucción y desarrollo de la lectura utilizando este medio (Blair y Rupley, 1986).

En los pocos estudios desarrollados, algunos de los investigadores e instituciones como los del Servicio Educacional de Pruebas (ETS) sugieren que la instrucción asistida por computador (IAC) pueden hacer que los aprendices o estudiantes aprendan en forma más rápida que la instrucción dada en una clase tradicional, mientras otros pregonan que los computadores no afectan ni la atención ni el aprendizaje. 
El Computador como Modo de Presentación de la Lectura en Inglés. Universidad de Nariño, Editorial Universitaria CEPUN. San Juan de Pasto. ISBN: 978-958-9479-12-4. 1998.

En estudios más recientes, la gente ha estado más preocupada por algunas de las implicaciones del uso de los computadores en la lectura (atención, terminación de un trabajo y comprensión). Belmore $(1983,1985)$ encontró que estudiantes de nivel universitario se demoraron más tiempo y comprendieron menos utilizando la lectura en el modo de presentación en el computador.

Zuk, (1986) trabajando con estudiantes más jóvenes también encontró que el efecto más importante del modo de presentación del texto en la lectura radicaba en el tiempo de terminación total de las tareas. Es decir, los sujetos se demoraron más al leer del computador que al hacerlo de la hoja de papel impresa. Sin embargo, no encontró diferencias significativas en cuanto a compresión de lectura.

También es conocida la especulación mercantilista, sobre todo a nivel internacional, acerca de los beneficios del uso de los computadores en educación desde hace ya dos décadas. Muchos cuestionamientos se han hecho acerca de las ventajas del uso de los computadores en el aula de clase (Kinzer, 1986), sin embargo hay muchas expectativas acerca del efecto real que estos puedan causar en cuanto a la atención y la motivación en los estudiantes (White, 1983)

En este nivel, y a pesar de que la investigación realizada es muy escasa y la que hay no es de tipo bien consistente, la mayor parte de la evidencia acerca de la utilidad de los computadores como una forma alterna de enseñanza y de aprendizaje en educación es de tipo anecdótico o personal. Y a pesar de que en la opinión de los expertos y según los resultados de algunas investigaciones pioneras se orientan a promover el uso de los computadores en la enseñanza de la lectura, muy pocos estudios se han realizado acerca de los efectos en cuanto al aprendizaje de la lengua (Blair y Rupley, 1986).

Benavides $(1993,1997)$ mediante un estudio similar, en el campo de la lectura y el vocabulario encontró que un grupo de estudiantes de nivel universitario mejoraron significativamente su nivel de velocidad y compresión de lectura en inglés como lengua extranjera mediante el uso de materiales auténticos en el modo de presentación tradicional, es decir, en papel. 
El Computador como Modo de Presentación de la Lectura en Inglés. Universidad de Nariño, Editorial Universitaria CEPUN. San Juan de Pasto. ISBN: 978-958-9479-12-4. 1998.

Sin embargo, en los trabajos mencionados anteriormente y en los que no ha habido unos resultados tan positivos como los esperados se discute que variables como la no suficiente familiarización con el medio electrónico en la presentación textual pudiera haber incidido en los mismos; o que el modo de pasar de una página a otra del texto o inclusive el hecho de no tener el texto mismo al alcance de la mano como sucede con el modo de presentación en papel hubiera podido ser determinante.

Según lo encontrado en el estudio de Hass y Hayes (1986) con estudiantes de nivel universitario que tenían experiencia en el manejo de textos escritos electrónicamente los sujetos pudieron ubicar frases más rápidamente en el texto escrito en papel que en la presentación electrónica del mismo en el computador. El tiempo promedio para ubicar una frase en el computador (32 segundos) fue significativamente superior al tiempo requerido para encontrar la misma en el texto impreso (13 segundos). Sin embargo estas diferencias disminuyeron al cambiar las características físicas de las pantallas del computador utilizadas.

\subsection{La lectura en ALAC}

ALAC se origina en CALL (Computer-Assisted Language Learning, por su sigla en inglés) Aprendizaje de Idiomas Asistido por Computador; o también definida como Informática Educativa para el Aprendizaje de los Idiomas, (INEDAI, Benavides 1993) o más recientemente como Aprendizaje de Lenguas Asistido por Computador (ALAC), que trata de la utilización de los computadores para suplementar el aprendizaje de los idiomas. Así que ALAC, en términos muy generales, puede entenderse como un concepto de utilizar el computador para propósitos de aprendizaje de lenguas, principalmente extranjeras. Este concepto plasmado en los diferentes tipos de software (programas) tiene como medio predominante la lectura a través de la pantalla del computador.

Podemos decir que ALAC comenzó como parte del proyecto educativo (PLATO) en los Estados Unidos, más específicamente en la Universidad de Illinois, uno de los sistemas más conocidos y de los proyectos educativos más ambiciosos que comenzaron en los 60's utilizando computadores centrales muy costosos para el aprendizaje de contenidos académicos. En 
El Computador como Modo de Presentación de la Lectura en Inglés. Universidad de Nariño, Editorial Universitaria CEPUN. San Juan de Pasto. ISBN: 978-958-9479-12-4. 1998.

Inglaterra los proyectos de tipo ALAC iniciaron durante la 70's uno de los cuales comenzó en la Universidad de Hull con bastantes expectativas.

Durante la década de los setenta con la llegada de microcomputadores económicamente más al alcance de la gente hubo muchos intentos, algunos exitosos, por introducir ALAC en instituciones educativas públicas y privadas. Sin embargo el desarrollo del hardware (la máquina) estaba muchísimo más adelantado que el de la cantidad y calidad (técnica y pedagógicamente hablando) del software disponible.

El auge de los microcomputadores en los años setenta y ochenta condujo a muchas instituciones a invertir en microcomputadores sin una planificación seria y sin base en necesidades concretas para lo que ellos querían hacer con estas máquinas. Este entusiasmo desbordado fue la regla en la mayoría de los países desarrollados con respecto al uso de este nuevo artefacto en aspectos educativos. Afortunadamente, la euforia descontrolada de la introducción de los computadores en educación parece haber disminuido a niveles más mesurados en estos días. A fin de cuentas, hay una lección para tenerse en cuenta y para aprender de lo que sucedió en varios países desarrollados sobre la introducción de los computadores en educación y en el aprendizaje de los idiomas, y es principalmente el hecho de que la introducción de la tecnología tiene que tomarse en forma más pragmática y tiene que ir de la mano de la pedagogía o algún tipo o enfoque de la misma.

Hoy ALAC se involucra con un tipo de actividades más comunicativas y la interacción usuariocomputador, y usuario-usuario se dice que es mucho más provechosa desde el punto de vista de la producción y más exigente a nivel cognitivo teniendo como medio de exposición e interacción la lectura. Sin embargo, ALAC ha alcanzado una etapa en la que la gente trata de encontrar un esquema pedagógicamente más sensato para explicar los diferentes procesos que pueden establecerse o lograrse por medio del computador: materiales informáticos, y consecuentemente los diferentes escenarios y contextos de aprendizaje. En otras palabras, se necesita comenzar a establecer una pedagogía de base que no tenga que relacionarse necesariamente con las teorías metodológicas existentes. 
El Computador como Modo de Presentación de la Lectura en Inglés. Universidad de Nariño, Editorial Universitaria CEPUN. San Juan de Pasto. ISBN: 978-958-9479-12-4. 1998.

Frecuentemente, las actividades en un contexto de ALAC, con el software o programas específicos, la máquina misma, para no mencionar la interacción lograda dentro de un ambiente de ALAC, han sido cuestionadas desde el punto de vista de su valor pedagógico. Sin embargo, han habido muchos esfuerzos (aunque aislados) para introducir, filtrar y poner a disposición de una gran gama de usuarios la nueva tecnología en contextos educativos con resultados prometedores. Esto es a veces ignorado por la mayoría de los críticos, o por parte de educacionalitas tecnófobos.

Según lo establecen Phillips (1985), y Weible (1987), (mencionados en Hubbard, 1992), ALAC y su tecnología han estado trabajando y buscando afanosamente una metodología apropiada y efectiva. A través de las diferentes etapas, los defensores de ALAC han propugnado por establecerlo como una disciplina por cuenta propia, y la abundante literatura apoya la necesidad de la apropiación de una estructura metodológica específica. Consecuentemente, se han realizado variados esfuerzos a fin de lograr esta meta. Higgins $(1983,1988)$. Underwood (1988), Phillips (1985), y Wyatt (1987), para mencionar solamente algunos, y más recientemente Hubbard, (1992) han surgido con algunas ideas de cómo pueden establecerse una estructura metodológica para ALAC en el campo de programas para cursos y desarrollo de materiales.

Como sucede en el campo de la instrucción en el aula de clases, no son los materiales, los elementos más importantes del proceso sino lo que el profesor y los estudiantes hacen con ellos, es decir el uso que se les da, pedagógicamente hablando. En el campo del Aprendizaje de Lenguas Asistido por Computador podríamos aplicar el mismo principio como fue el caso de los comienzos de ALAC en los que el computador solo fue utilizado como parte de materiales audiovisuales que ayudaba al profesor en las labores de instrucción.

\subsection{La lectura y los multimedios.}

Los multimedios (MULTIMEDIA) es uno de los últimos desarrollos en la tecnología del computador y se basa en el concepto de la integración de sonido (desde la voz humana incluida, hasta sonido estéreo de alta calidad), video, gráficos y texto. Este tipo de medios diferentes y sin embargo complementarios se obtienen a partir de discos compactos con tecnología láser 
El Computador como Modo de Presentación de la Lectura en Inglés. Universidad de Nariño, Editorial Universitaria CEPUN. San Juan de Pasto. ISBN: 978-958-9479-12-4. 1998.

cuya base es un computador de tipo medio equipado con una unidad de disco compacto (CD ROM). Estos pueden almacenar una enciclopedia de la lengua completa como parte de la información textual.

Este nuevo desarrollo en la tecnología del computador presenta tanto a profesores como a estudiantes una amplia gama de oportunidades tanto desde el punto de vista de la enseñanza magistral como del aprendizaje implícito. Sin embargo, este tipo de recursos no están aún al alcance de todas las instituciones educativas de nivel básico primario o secundario. Por otro lado todavía existe incertidumbre de cuán efectivo sea, este tipo de tecnología para que pueda contribuir realmente en el mejoramiento del aprendizaje de una lengua, sobre todo en lo que atañe a la lectura.

Hay por supuesto muchas otras posibilidades para el aprendizaje de la lectura y el vocabulario con tipos diferentes de programas especialmente con aquellos que utilizan los Multimedios (Multimedia) donde la integración de sonidos, videos, imágenes, y texto parece proveer oportunidades más ricas e interactivas para aprender. Consecuentemente, se piensa que este tipo de actividades recreadas por medio del computador podría desarrollar diferentes estilos de aprendizaje, el de la lectura y el vocabulario entre ellos.

\subsection{La lectura y la Red Mundial de la Información (INTERNET)}

A pesar de la introducción de la Multimedia en la Red Mundial, esta es todavía predominantemente un océano de palabras y texto siendo el inglés el idioma de comunicación por excelencia. La precisión, claridad y buen manejo de la ortografía son muy importantes para la comunicación electrónica efectiva.

Precisamente, en este entorno se destaca la lectura, ya que una persona que no pueda leer en inglés o cuyas habilidades de lectura sean limitadas prácticamente su aprovechamiento de la información en la Red van estar considerablemente afectadas. Las habilidades de la comprensión, interpretación, síntesis de la información, skimming (lectura rápida) y scanning (búsqueda de información particular) son bastante necesarias para manejar y procesar el texto: información, y mensajes recibidos desde una muy amplia gama de fuentes. Ahora, no solo hay 
El Computador como Modo de Presentación de la Lectura en Inglés. Universidad de Nariño, Editorial Universitaria CEPUN. San Juan de Pasto. ISBN: 978-958-9479-12-4. 1998.

que saber cómo hacerlo, es decir el uso del conocimiento, sino hacerlo rápido ya que en el caso la conexión con la Red Mundial, el tiempo de conexión cuenta importantemente.

La Red Mundial está atrayendo cada día más casas editoriales como el caso de The Guardian y The Electronic Telegraph los cuales están disponibles en línea. Otro caso para el progresivo dominio del texto en inglés es el servidor Gutemberg que contiene más de 2000 texto listos para ser inspeccionados, leídos o inclusive copiados y compartidos ya que están exentos de derechos de copia.

El idioma inglés como medio principal de comunicación de la Red Mundial está de alguna manera experimentando un proceso de total desarrollo debido a su uso diario a nivel mundial. Muchos inclusive han llegado a pensar que su importancia y dominio crecerá y prevalecerá puesto que está creando una verdadera área de estudio en este medio y una forma de recoger información acerca de cualquier tema en el mundo entero y compartirlo es hacerlo accesible en la Red. Esto está siendo replicado cada vez más por miles de personas en un ciclo interminable del proceso de la información.

Los estudiantes probablemente estarán muy pronto utilizando bibliotecas multimediales en línea, lo que les proporcionará muchas posibilidades para aprender y realizar investigación en diferentes campos. De la misma forma estarán utilizando habilidades sofisticadas para el manejo de la información (seleccionar, evaluar, sintetizar, etc.) que de por sí ya implica comprensión, producción y difusión del mensaje y estos tres componentes se realizan más rápidamente y con mayor intensidad que en épocas anteriores.

Los estudiantes estarán, en forma eficaz y colaborativa, intercambiando, revisando, refinando y publicando sus trabajos para ponerlos a disposición de la vasta audiencia de la Red Mundial, trabajos estos que posiblemente no serán producidos por uno solo sino por muchos a la vez. Estos trabajos muy probablemente con niveles de lectura adecuados para su compresión no serán completamente terminados. Textos mixtos podrían emerger con secciones fijas en Disco óptico o papel, y por supuesto hipertexto de evolución continua en INTERNET donde la lectura en inglés cobrará un papel de innegable importancia. 
El Computador como Modo de Presentación de la Lectura en Inglés. Universidad de Nariño, Editorial Universitaria CEPUN. San Juan de Pasto. ISBN: 978-958-9479-12-4. 1998.

\section{Bibliografía}

Belmore, S. (1985). Reading Computer-presented Text. Bulletin of the Psychonomic Society. 23(1), 12-24.

Benavides B., J. E. (1989). Iniciación a la Instrucción Complementaria del Inglés Mediante Computadores. Revista de Investigaciones, Universidad de Nariño, 3(5), 179-193.

Benavides B., J. E. (1993). Informática Educativa para el Aprendizaje de los Idiomas. Revista de Investigaciones, Universidad de Nariño, 6(1), 196-206.

Benavides B., J. E. (1994). Desarrollo de la Velocidad de Lectura y Aprendizaje de Vocabulario en Estudiantes de último semestre de Lenguas Modernas de la Universidad Mariana. Revista Criterios, Universidad Mariana, 1(2), 38-58.

Benavides B., J. E. (1997). La Lectura Rápida en el Desarrollo de la Velocidad y la Comprensión en Inglés: desarrollo de la Lectura, Velocidad, Comprensión, Vocabulario y Gramática es Estudiantes de Inglés Técnico a Nivel Universitario. San Juan de Pasto: Universidad de Nariño - CEPUN.

Benavides B., J. E. (1998). El Computador en el Desarrollo de la Lectura en Inglés. San Juan de Pasto: Centro de Investigaciones, Universidad Mariana. La Hormiga.

Blair, F. L., Rupley, W. \& Jones, M (1986). Microcomputers: Another False Prophet? Reading Research and Instruction. 26(1), 58-61.

Henao, O. \& Giraldo, L. (1991). El Computador en la Enseñanza de la Escritura. Medellín: (Universidad de Antioquia): Textos y Diseños.

Johnson, La mont. (1986). The Three Phases of Educational Computing. Computers in the Schools. 3(2), 1-2.

Kinzer, Ch. (1986). A Five Part Categorization for Use of Microcomputers in Reading Classrooms. Journal of Reading. 30(3), 56-76.

Kolers, P. Duchnieky, R. \& Ferguson, D. (1981). Eye Movement Measurement of readabiblity of CRT Displays. Human Factors. 23(5), 517-527.

Lewis, G. \& B. del Castillo. (1993). English through Computers (1 \& 2). Madrid: McGraw-Hill.

Mourant, R. Lakshmanan, R. \& Chantadisal, R. (1981). Visual Fatigue and Cathode Ray Tube Display Terminal. Human Factors. 23(5), 529-540.

Nuttal, C. 1985. Teaching Reading Skills in Another Language. London: Heinemann Educational Books.

Smith, F. (1982). Understanding Reading. New York: Holt, Rinehart and Winston.

White, M. 1983. Synthesis of Research on Electronic Learning. Educational Leadership. 40(8), 1315.

Zuk, D. (1986). The Effects of Microcomputers on Children's Attention to Reading. Computers in the Schools. (3), 39-51. 